Angelo Marino was born in Napoli, Italy, on May 5, 1940. He received the $\mathrm{Ph} . \mathrm{D}$. degree in physics from the University of Rome, Rome, Italy. $\mathrm{He}$ has worked on photonuclear interactions and Compton backscattering on high energy electrons. Since 1975 his research has been in high power excimer lasers and more recently, since 1977, in FEL devices. He is Group Leader of the FEL CNEN-Frascati Project at CNEN, Rome, Italy.
Alberto Renieri was born in Certaldo, Florence, Italy, on August 11, 1945. He received the Ph.D. degree in physics from the University of Rome, Italy, in 1969, with a dissertation on bunched beam instabilities in electron storage ring accelerators.

Since 1971 he has been a staff member of the CNEN Frascati Center, Rome, Italy. From 1971 to 1976 he worked on theoretical problems connected with particles dynamics in high energy electron accelerators. He joined the CNEN Laser Division in 1976 where he is currently concerned with quantum optics theory and, in particular, free-electron laser theory.
Francesco Romanelli was born in Florence, Italy, on April 26, 1956. $\mathrm{He}$ received the Ph.D. degree in physics from the University of Florence in 1980 .

Since 1979 he has been with the CNEN Frascati Center, Rome, Italy, initially as a student and then as a Guest Researcher working on the coherence properties and the multimode theory of FEL. He is presently a CNEN Fellow.

\title{
Inclusion of Space-Charge Effects with Maxwell's Equations in the Single-Particle Analysis of Free-Electron Lasers
}

\author{
CHUN-CHING SHIH AND AMNON YARIV, FELLOW, IEEE
}

\begin{abstract}
In free-electron lasers, the motion of electrons is governed by the Lorentz force equations in the presence of a radiation field. The variation of the radiation field then follows from Maxwell equations in which the modulated electron current acts as a source. A self-consistent analysis, which completely incorporates these two concepts, is presented to describe the behavior of the radiation field and the modulation of the electron beam. In this analytical study, we consider the space-charge effect as well as high interaction strength in the smallsignal limit so that the result can be compared directly with the travelingwave tube theory. It is found that the well-known three-wave solution is essentially applicable to the electron beam only. The variation of the radiation field is much more complicated. According to the analysis, there are only three controlling parameters: the pumping strength, the electron density, and the electron energy detuning. For different choices of those parameters, the field can be in a stable regime where its growth is limited or in an unstable regime where it grows exponen-
\end{abstract}

Manuscript received January 6, 1981; revised March 26, 1981. This work was supported by the U.S. Air Force Office of Scientific Research.

C.-C. Shih was with the Steele Laboratory, California Institute of Technology, Pasadena, CA 91125. He is now with TRW, Redondo Beach, CA 90278.

A. Yariv is with Steele Laboratory, California Institute of Technology, Pasadena, CA 91125. tially. The boundary between these two regimes is defined quantitatively. The effect of the plasma resonance is observed at high electron densities as a natural result of maximum single-pass gains. The travelingwave tube is then analyzed as a special example.

\section{INTRODUCTION}

T $\mathrm{N}$ the free-electron laser, two physical quantities are of concern: the electron beam and the electromagnetic field. Theoretical studies describe their evolution based on proper initial conditions and controlling interaction equations. The electron beam, in general, is treated by either the single-particle picture or the use of the electron distribution function. In the latter case, the evolution of the distribution function is studied from the Boltzmann equation [1]. In the former case, the trajectory of the individual electron in real space or phase space is obtained from the Lorentz force equation [2], [3] or, equivalently, the single-particle Hamiltonian including field vector potentials [4], [5]. The ensemble average of all quantities over the initial electron states is necessary to obtain the final working results. 
To calculate the change of the radiation field, the process can be simplified when the amount of energy exchanged is negligible compared to the input radiation energy. The radiation field is then assumed to have a nearly constant amplitude and phase. The small energy increase in radiation is obtained from the computed energy loss of the electron beam. This approximation has been used extensively and is suitable for a high $Q$ (low gain) device. However, if the device is a signal amplifier or a low $Q$ oscillator, the change in radiation becomes significant along the interaction region. In this case, it becomes necessary to account for the radiation field selfconsistently, which can be achieved by considering Maxwell's equations self-consistently with the Boltzmann equation. This leads to a dispersion equation for the wavenumber of the propagating field $[6]-[8]$. The real part of the wavenumber yields the phase change, while its imaginary part represents the amplitude variation. The similarity in gain between freeelectron lasers and traveling-wave tubes has thus been demonstrated [6].

Not until recently has the self-consistent treatment been employed in the single-particle model [9], [10]. In this paper we will show how these two concepts can be incorporated. In order to present the idea analytically, we have limited our analysis to the small-signal regime. The result may not be correct for a high-power device in steady state, but it describes properly how the field grows from the noise. Therefore, the result might determine if oscillation is possible under certain conditions. For simplification, other conditions are idealized. For example, we assume that the electron beam is monoenergetic and collimated, and the radiation field is a plane wave. But we intend to include the space-charge effect in the strong interaction regime so that the result can be compared directly with the traveling-wave tube theory [11].

In Section II, a set of coupled equations describing the interrelations between the electron beam and the radiation field are derived from the Lorentz and Maxwell equations. In Section III, the coupled equations are solved. Different types of solutions are examined, and their operational ranges are quantitatively defined. In the last section, the behavior of the radiation field is shown explicitly. A special example, which resembles a traveling-wave tube, is discussed in detail. Conclusions are then drawn.

\section{THE COUPLed Equations}

The interaction between an electron and the radiation is described by the Lorentz force equations

$$
\begin{aligned}
& \frac{d}{d t}(m \gamma \vec{v})=e\left(\vec{E}+\frac{1}{c} \vec{v} \times \vec{B}\right) \\
& \frac{d}{d t}\left(\gamma m c^{2}\right)=e \vec{v} \cdot \vec{E}
\end{aligned}
$$

where $\vec{v}$ and $\gamma m c^{2}$ are the velocity and energy of the electron, and $\vec{E}$ and $\vec{B}$ are the total electric and magnetic field in the interaction region.

The electron beam propagates in the $z$ direction and through the axis of a uniform helical magnet where the magnetic field is given by

$$
\vec{B}_{m}=B\left(\cos \frac{2 \pi z}{l},-\sin \frac{2 \pi z}{l}, 0\right) .
$$

The spontaneous and stimulated radiation fields in this magnet are circularly polarized. Neglecting all dependence on transverse variables, the radiation field is represented by

$$
\begin{aligned}
& \vec{E}_{r}=E(t)(\cos (\omega t-k z+\phi+\psi(t)), \\
&- \sin (\omega t-k z+\phi+\psi(t), \\
& \vec{B}_{r}=E(t)(\sin (\omega t-k z+\phi+\psi(t)), \\
& \cos (\omega t-k z+\phi+\psi(t)),
\end{aligned}
$$

where $\omega=k c$ is the radiation frequency, $E(t)$ is the field strength, $\phi$ is the phase at the entrance of the interaction region, and $\psi(t)$ is the possible phase variation with the initial condition $\psi(0)=0$. For convenience, we have written $E$ and $\psi$ as functions of time $t$ instead of position $z$. In the smallsignal limit, this does not make any difference.

The longitudinal space-charge field $E_{c z}$ can be found from the Poisson equation

$$
\frac{\partial}{\partial z} E_{c z}(z, t)=4 \pi e\left[N(z, t)-N_{0}\right]
$$

where $N(z, t)$ is the electron density at position $z$ and time $t$, and $N_{0}$ is the initial electron density. Using (4), it has been shown that the space-charge field can be related to the dynamic variable of an electron as [12]

$$
\begin{aligned}
E_{c z}(z, t) & =-4 \pi e N_{0}\left[\Delta z(\phi, t)-\langle\Delta z(\phi, t)\rangle_{\phi}\right] \\
\Delta z(\phi, t) & =z(\phi, t)-v_{0} t
\end{aligned}
$$

where $\Delta z$ is the position modulation of the electron. $v_{0}$ is the electron initial velocity and \langle\rangle$_{\phi}$ is the ensemble average over the initial phase distribution. To obtain (5), it is assumed that the electron beam behaves as a "single stream." This assumption requires that the relative position of any two electrons cannot be reversed and is true only in the small-signal limit.

The total field in (1) is then $\vec{E}=\vec{E}_{r}+\vec{E}_{c z}$ and $\vec{B}=\vec{B}_{r}+\vec{B}_{m}$. The Lorentz force equations are reduced to a one-dimensional equation describing the parallel motion only [12]:

$$
\begin{aligned}
\frac{d^{2}}{d t^{2}} & \Delta z(t)+\omega_{p}^{2}\left[\Delta z(t)-\langle\Delta z(t)\rangle_{\phi}\right] \\
& =a E(t) \cos [\Omega t-\beta \Delta z(t)+\phi+\psi(t)] \\
a & =\frac{2 e^{2} B}{\gamma^{2} m^{2} c \omega} \\
\Omega & =\omega-\beta v_{0} \\
\beta & =k+\frac{2 \pi}{l} \\
\omega_{p}^{2} & =\frac{4 \pi e^{2} N_{0}}{m \gamma^{3}}
\end{aligned}
$$

where $a$ is the interaction strength due to the static magnetic field and $\Omega$ is the off-resonance parameter. $\beta$ is the wavenumber of the first harmonic of the radiation in a periodic structure with period $l$ and $\omega_{p}$ is the longitudinal relativistic plasma frequency. In (6), we have assumed that the particle is nearly 
in-resonance with the interaction field $\Omega \simeq 0$, and the percentage change of $E$ and $\psi$ is very small over a magnet period, i.e.,

$$
\begin{aligned}
& \frac{d}{d t} E(t)<<\frac{c}{l} E(t) \\
& \frac{d}{d t} \psi(t)<<\frac{c}{l} \psi(t) .
\end{aligned}
$$

For the case of constant $E$ and $\psi,(6)$ has been solved for $\Delta z(t)$ in the small-signal limit [11]. A space charge gain is obtained which displays gain saturation and the plasma resonance at high electron densities. Since, in general, $E(t)$ and $\psi(t)$ are unknown function of time, $\Delta z(t)$ cannot be obtained directly in the high-gain situation. However, it is appropriate to assume $\Delta z(t)$ in the form

$$
\Delta z(t)=a E(0)[f(t) \cos \phi+g(t) \sin \phi]
$$

where $E(0)$ is the initial field amplitude and works as a perturbation constant in the small-signal limit. $f(t)$ and $g(t)$, which are independent of $\phi$, represent the cosine and sine modulations of the electron beam. Substituting (8) into (6), we have

$$
\begin{aligned}
\frac{d^{2}}{d t^{2}} f(t)+\omega_{p}^{2} f(t) & =\tilde{E}(t) \cos \alpha(t) \\
\frac{d^{2}}{d t^{2}} g(t)+\omega_{p}^{2} g(t) & =-\tilde{E}(t) \sin \alpha(t) \\
\alpha(t) & =\psi(t)+\Omega t ; \quad \tilde{E}(t)=E(t) / E(0)
\end{aligned}
$$

where $\tilde{E}(t)$ is the normalized field amplitude, i.e., $\tilde{E}(0)=1$.

Since, due to the interaction with the radiation, the electron beam is no longer uniform, the modulated current will generate a transverse field according to the transverse Maxwell equation

$$
\left(\frac{\partial^{2}}{\partial z^{2}}-\frac{1}{c^{2}} \frac{\partial^{2}}{\partial t^{2}}\right) A_{\perp}(z, t)=-\frac{4 \pi}{c} J_{\perp}(z, t)
$$

where the transverse vector potential is related to the radiation field as

$$
\vec{E}_{r}(z, t)=\frac{1}{c} \frac{\partial}{\partial t} A_{\perp}(z, t) .
$$

The transverse current is expressed as

$$
J_{\perp}(z, t)=e N_{0} v_{\perp}(z, t)
$$

where the transverse velocity $v_{\perp}$ can be obtained from the integration of the transverse component of the Lorentz force equation (1):

$$
\begin{aligned}
\boldsymbol{v}_{\perp}= & -\frac{e B l}{2 \pi m c \gamma}\left(\cos \frac{2 \pi z}{l},-\sin \frac{2 \pi z}{l}, 0\right) \\
& +\frac{e E}{m \gamma \omega}(\sin (\omega t-k z+\phi+\psi(t)), \\
& \cos (\omega t-k z+\phi+\psi(t)), 0) .
\end{aligned}
$$

Using the facts that $E(t)$ and $\psi(t)$ are slowly varying functions and

$$
\frac{d}{d t} \equiv \frac{\partial}{\partial z}+\frac{1}{c} \frac{\partial}{\partial t},
$$

(10) becomes

$$
\begin{aligned}
\frac{d}{d t} E(t) \hat{\epsilon}_{1}+E(t) & \frac{d}{d t} \psi(t) \hat{\epsilon}_{2}=-\frac{4 \pi}{c} J_{\perp}(z, t) \\
\hat{\epsilon}_{1} & \equiv \vec{E}(t) / E(t) ; \quad \hat{\epsilon}_{2} \equiv \vec{B}(t) / E(t)
\end{aligned}
$$

where $\hat{\epsilon}_{1}$ and $\hat{\epsilon}_{2}$ are the unit vectors in the direction of the electric and magnetic component of the radiation field. Since these two unit vectors are orthogonal to each other, we have

$$
\begin{aligned}
\frac{d}{d t} E(t) & =2 \pi N_{0} e\left\langle v_{\perp} \cdot \hat{\epsilon}_{1}\right\rangle_{\phi} \\
E(t) \frac{d}{d t} \psi(t) & =-2 \pi N_{0} e\left\langle v_{\perp} \cdot \hat{\epsilon}_{2}\right\rangle_{\phi} .
\end{aligned}
$$

We have taken ensemble average in (16) because the left side varies in a scale much longer than $l / c$. Substituting (3), (8), and (14) into (16), we obtain two equations

$$
\begin{aligned}
\frac{d}{d t} \tilde{E}(t) & =b[f(t) \sin \alpha(t)+g(t) \cos \alpha(t)] \\
\tilde{E}(t) \frac{d}{d t} \alpha(t) & =b[f(t) \cos \alpha(t)-g(t) \sin \alpha(t)]+\Omega^{\prime} \tilde{E}(t) \\
b & =\frac{e^{2} B^{2} l \omega_{p}^{2}}{4 \pi m^{2} c^{3}} \\
\Omega^{\prime} & =\Omega+\frac{\gamma^{2} \omega_{p}^{2}}{2 \omega} .
\end{aligned}
$$

We have to point out that we have neglected in (13) the terms including $d E(t) / d t$ and $d \psi(t) / d t$. These terms, after the phase average in (16), result in a correction factor $\left[1+\left(\gamma^{2} \omega_{p}^{2} /\right.\right.$ $\left.2 \omega^{2}\right)^{-1}$ ] for $b$. Since $\left(\gamma^{2} \omega_{p}^{2} / 2 \omega^{2}\right)$ is always much smaller than unity, we neglect the correction. Combining (9) and (17) results in a set of four coupled equations:

$$
\begin{aligned}
\frac{d^{2}}{d t^{2}} f+\omega_{p}^{2} f & =\tilde{E} \cos \alpha \\
\frac{d^{2}}{d t^{2}} g+\omega_{p}^{2} g & =-\tilde{E} \sin \alpha \\
\frac{d}{d t} \tilde{E} & =b[f \sin \alpha+g \cos \alpha] \\
\tilde{E} \frac{d}{d t} \alpha & =b[f \cos \alpha-g \sin \alpha]+\Omega^{\prime} \tilde{E}
\end{aligned}
$$

which show how the electron modulation is coupled to the field variation.

\section{SELF-CONSISTENT SOlutions}

In the last section, we obtained a set of coupled equations describing the interrelations between the electron modulation and the field variation. The final purpose of the analysis is to obtain the behavior of the normalized radiation amplitude $\widetilde{E}(t)$. Based on (18)-(21), we find it impossible to derive an 
equation for $E(t)$ only. The decoupling of those four equations results in a sixth-order simple differential equation for the modulation functions $f(t)$ and $g(t)$. To simplify the process, we choose a dimensionless variable

$$
\tau=t / T
$$

and three dimensionless parameters

$$
\begin{aligned}
\theta_{p} & =\omega_{p} T \\
\theta & =\Omega^{\prime} T \\
P & =b^{1 / 3} T
\end{aligned}
$$

where $T=L / c$, and $L$ is the device length.

A differentiation of (18) and (19) leads to the following coupled equations:

$$
\begin{aligned}
& \frac{d^{3} f}{d \tau^{3}}+\theta_{p}^{2} \frac{d f}{d \tau}=\theta \frac{d^{2} g}{d \tau^{2}}+\left(P^{3}+\theta \theta_{p}^{2}\right) g \\
& \frac{d^{3} g}{d \tau^{3}}+\theta_{p}^{2} \frac{d g}{d \tau}=-\theta \frac{d^{2} f}{d \tau^{2}}-\left(P^{3}+\theta \theta_{p}^{2}\right) f
\end{aligned}
$$

which result finally in a differential equation

$$
\begin{aligned}
& \frac{d^{6} h}{d \tau^{6}}+\left(2 \theta_{p}^{2}+\theta^{2}\right) \frac{d^{4} h}{d \tau^{4}}+\left(\theta_{p}^{4}+2 P^{3} \theta\right) \frac{d^{2} h}{d \tau^{2}}+P^{6} h=0 \\
& h \equiv f \text { or } g .
\end{aligned}
$$

Once $f(t)$ and $g(t)$ are found, the gain can be obtained from equation (20) directly as

$$
G(t) \equiv \tilde{E}(t)-1=2 b\left[\frac{d f}{d t} g-f \frac{d g}{d t}\right] .
$$

Therefore, the problem is reduced to finding solutions of the sixth-order differential equation (25) which is controlled completely by the three parameters given in (23). The constant $\theta_{p}$ measures the contribution of the space-charge effect. The constant $\theta$ indicates the detuning of the monoenergetic electron beam. The constant $P$ represents the interaction strength which is proportional to the square of the magnetic field. Although $b$ includes a factor $\omega_{p}^{2}$, it indicates only the number of electrons participating in the interaction and has nothing to do with the space-charge effect. Thus, we intend not to write out the factor explicitly in order to distinguish it from the plasma frequency appearing in $\theta_{p}$.

Since all coefficients in (25) are constant, the most general solutions for $f(t)$ and $g(t)$ are in exponential forms

$$
f=A e^{i \Gamma \tau} ; \quad g=B e^{i \Gamma \tau} .
$$

Using (27), (25) becomes

$$
\left(\Gamma^{3}+\theta_{p}^{2} \Gamma\right)^{2}+\left(\theta \Gamma^{2}+P^{3}+\theta \theta_{p}^{2}\right)^{2}=0 .
$$

The general solutions will be

$$
f(t)=\sum_{n=1}^{6} A_{n} e^{i \Gamma_{n} \tau} ; \quad g(t)=\sum_{n=1}^{6} B_{n} e^{i \Gamma_{n} \tau}
$$

where $\Gamma_{n}(n=1,6)$ are the solutions of $(28) . A_{n}$ and $B_{n}$ are coefficients which are related to each other by (27) and determined by proper initial conditions

$$
\begin{aligned}
f(0) & =0 ; & g(0)=0 \\
\frac{d}{d t} f(0) & =0 ; & \frac{d}{d t} g(0)=0 \\
\frac{d^{2}}{d t^{2}} f(0) & =1 ; & \frac{d^{2}}{d t^{2}} g(0)=0 .
\end{aligned}
$$

The first pair of initial conditions come from $\Delta z(0)=0$, while the second pair comes from $\Delta v(0)=0$. The last two conditions are observed directly from (18) and (19).

Before we find out the solutions explicitly, it is interesting to determine what kind of solutions are possible.

From (28) it can be seen that the solutions are in three conjugated pairs since all coefficients are real. Second, if $\Gamma$ is a solution of $(28),(-\Gamma)$ must also be a solution. Third, $\Gamma$ cannot be real because (28) cannot be satisfied for all real solutions. Based on these three basic properties, we conclude that there are only two possible types of solutions.

Type (A): All are imaginary:

$$
\Gamma= \pm i u ; \quad \pm i v ; \quad \pm i w .
$$

Type (B): Two are imaginary and four are complex:

$$
\Gamma= \pm i u ; \quad \pm r \pm \text { is. }
$$

These two types of solutions reveal the feature of three waves which is well known in the traveling-wave tube theory. However, we discover that the three-wave property applies essentially to the electron beam modulation and not the radiation field. According to (26), the behavior of the radiation field is much more complicated than a mere three-wave propagation.

Equation (28) can also be reduced to a conjugated pair of third-order differential equations with complex coefficients

$$
\Gamma^{3} \pm i \theta \Gamma^{2}+\theta_{p}^{2} \Gamma \pm i\left(P^{3}+\theta \theta_{p}^{2}\right)=0 .
$$

This equation can be shown to be equivalent to the dispersion equation to some extent. Assume the solutions of (33) are $\Gamma_{1}, \Gamma_{2}, \Gamma_{3}$. From equation (26), the radiation intensity behaves as

$$
\tilde{E}^{2}(t) \sim e^{\chi \tau}
$$

where $\chi$ must be the sum of any two $\Gamma_{i}$. If we pick

$$
\chi_{1}=\Gamma_{2}+\Gamma_{3} ; \quad \chi_{2}=\Gamma_{3}+\Gamma_{1} ; \quad \chi_{3}=\Gamma_{1}+\Gamma_{2},
$$

the equation for $\chi$ can be derived from the relations of coefficients. The new equation is

$$
\chi\left[\chi+i\left(\theta-\theta_{p}\right)\right]\left[\chi+i\left(\theta-\theta_{p}\right)\right]+i P^{3}=0 .
$$

This is exactly the dispersion equation for the radiation field [13]. However, the combinations in (35) are far from sufficient. In general, $\chi$ must be the combination of the six solutions of (28) instead the three solutions of (33). Therefore, it includes the combinations such as $\left(\Gamma_{1}+\Gamma_{2}^{*}\right)$. The field is then more complicated than the three waves described by (36).

From (31) and (32), it is understood that the type A solutions are interference-like while the type $\mathbf{B}$ solutions are exponential-like. If the parameters are chosen such that the solutions are of type $\mathrm{A}$, the field has its maximum and cannot grow any further even for a longer magnet. It is thus im- 


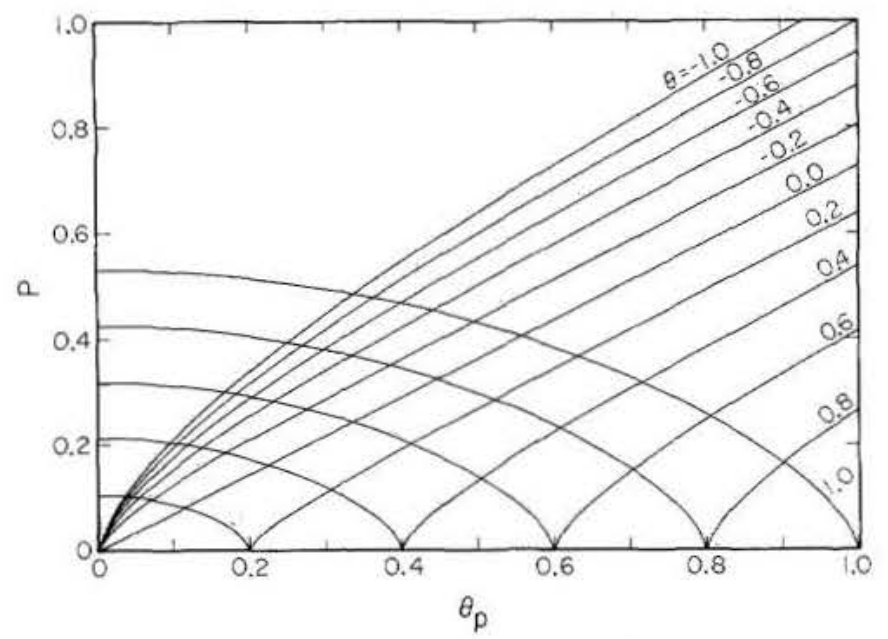

Fig. 1. Plot of boundary between the stable (below) and unstable (above) regions for constant $\theta$.

portant to find out the conditions which distinguish one type of solutions from the other. The result serves to determine if the stimulated radiation can grow from the noise in an oscillator.

Let

$$
\Gamma=i \delta \text {. }
$$

We have

$$
\delta^{3}+\theta \delta^{2}-\theta_{p}^{2} \delta-\left(P^{3}+\theta \theta_{p}^{2}\right)=0
$$

where only the equation in (33) with positive signs is considered.

The solutions of $(38)$ must be $(u, v, w)$ or $(u, s \pm i r)$ and are determined by the secular equation for a three-power polynomial equation:

$$
D \equiv 4\left(\frac{\theta^{2}}{3}+\theta_{p}^{2}\right)^{3}-27\left(P^{3}-\frac{2}{27} \theta^{3}+\frac{2}{3} \theta \theta_{p}^{2}\right)^{2} .
$$

The condition $D=0$ gives

$$
P=\frac{1}{3}\left[18 \theta \theta_{p}^{2}-2 \theta^{2}+2\left(3 \theta_{p}^{2}+\theta^{2}\right)^{3 / 2}\right]^{1 / 3}
$$

which describes a surface in the space $\left(\theta, \theta_{p}, P\right)$ separating those two types of solutions. In (40), we have discarded the other possible surface [negative sign in front of 2()$^{3 / 2}$ ] because it is in the negative half-space of $\boldsymbol{P}$ which is unphysical. To show the surface at different positions, (40) is plotted for constant $\theta$ (Fig. 1) and $\theta_{p}$ (Fig. 2). The region of unstable solutions occupies the space above the curve, while the stable solutions occupy the lower space.

Although the curves are shown only for parameter values up to unity, it is interesting to note that the coordinates and the constant can be scaled up or down simultaneously with the same factor. This property gives a convenient way for constructing the three-dimensional surface by the spherical coordinates $(R, \eta, \xi)$ defined by

$$
\begin{aligned}
\theta & =R \sin \eta \cos \xi \\
\theta_{p} & =R \sin \eta \sin \xi \\
P & =R \cos \eta .
\end{aligned}
$$

The surface is thus described by

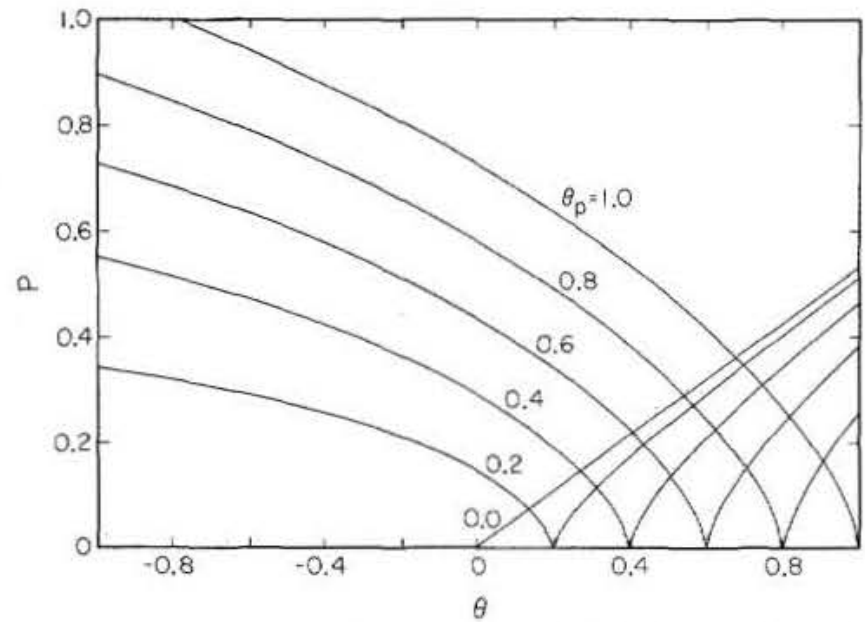

Fig. 2. Plot of boundary between the stable (below) and unstable (above) regions for constant $\theta_{p}$.

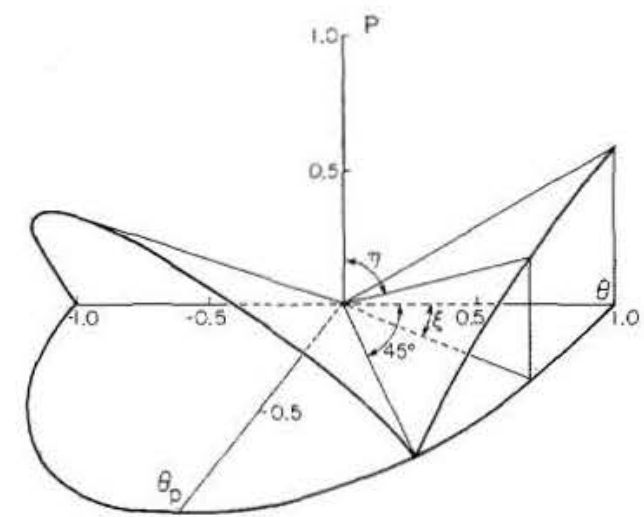

Fig. 3. Three-dimensional plot of the boundary. The valley around $\theta=\theta_{p}$ is responsible for the plasma resonance at high electron densities.

$$
\cot \eta=\frac{1}{3}\left[\cos \xi\left(20 \cos ^{2} \xi-18\right)+2\left(3-2 \cos ^{2} \xi\right)^{3 / 2}\right]^{1 / 3}
$$

and is independent of the distance from the origin $R$ (Fig. 3). This surface defines the threshold pumping strength for an exponentially growing field. The value drops dramatically to zero at $\theta=\theta_{p}$, which is known as a condition for plasma resonance [12]. However, an unstable solution may not work better than a stable solution for a finite interaction length. As shown in [8], the stable solution leads to a better gain for the example considered there. Therefore, the choice of unstable solutions may not be favorable unless the interaction length is long enough. For a typical example where $\gamma=50, \lambda=10.6 \mu \mathrm{m}$, $\omega_{p} \gamma^{3 / 2}=10^{10} \mathrm{~s}^{-1}, B=3 \mathrm{kG}$, and $L=3 \mathrm{~m}$, we find $\theta_{p} \simeq 0.4$ and $P \simeq 3$. The system is in the unstable region when $\theta<6$.

The three-dimensional plot provides a convenient method for locating the system in an amplifier where all three parameters are independent of each other. For an oscillator, only $P$ and $\theta_{p}$ are independent parameters. The radiation frequency (and thus $\theta$ ) adjusts itself automatically until the single-pass gain is maximum. In the low gain limit, the behavior of $\theta$ has been shown explicitly as the electron density varies [12]. The value of $\theta$ is constant $(\simeq 2.6)$ for small $\theta_{p}$ and approaches $\theta_{p}$ at large values of $\theta_{p}$ (see [12, Fig. 4]). 
For large interaction strength, the behavior of $\theta$ is somehow complicated by parameter $P$. To observe quantitatively the behavior of $\theta$ in the unstable region, we have to locate the maximum exponential constant and thus the imaginary part of the solutions. From the theory of polynomial equations, the imaginary part is found to be

$$
\operatorname{Im}=\frac{\sqrt{3}}{\sqrt[3]{2}} \sinh \frac{M}{3}
$$

where

$$
\cosh ^{2} M=\frac{\left(27 P^{3}+18 \theta \theta_{p}^{2}-2 \theta^{3}\right)^{2}}{4\left(\theta^{2}+3 \theta_{p}^{2}\right)^{3}} .
$$

By taking

$$
\left.\frac{\partial \mathrm{Im}}{\partial \theta}\right|_{\theta_{p}, P}=0
$$

it is possible to find the optimum value of $\theta$ for given $\theta_{p}$ and $P$. However, we are especially interested in what happens at high electron densities and long interaction length. At these limits, the behavior of $\theta$ can be understood qualitatively from Fig. 3. The surface remains the same if we scale down all three coordinates with the factor $\theta_{p}$. The new coordinates are

$$
\left\{\begin{array}{l}
x=\theta / \theta_{p} \\
y=1 \\
z=P / \theta_{p} \propto \theta_{p}^{-1 / 3} .
\end{array}\right.
$$

The system stays at $y=1$, but $z$ becomes smaller as $\theta_{p}$ increases. Since unstable solutions are favorable for long interaction length, the system remains in the valley and approaches to the bottom where $x=y$. This implies plasma resonance $\theta=\theta_{p}$ at high $\theta_{p}$.

To obtain gain behaviors, we have to first solve (25) for $f(t)$ and $g(t)$. The algebra is straightforward but tedious. The results for both types of solutions are shown in the following.

Type A:

$$
\begin{aligned}
f(t)= & \frac{\cos u \tau}{(u-v)(w-u)}+\frac{\cos v \tau}{(v-w)(u-v)}+\frac{\cos w \tau}{(w-u)(v-w)} T^{2} \\
g(t)= & \frac{\sin u \tau}{(u-v)(u-w)}+\frac{\sin v \tau}{(v-w)(v-u)}+\frac{\sin w \tau}{(w-u)(w-v)} T^{2} \\
G(t)= & \frac{2 P}{(u-v)(v-w)(w-u)}\left\{\frac{u+v}{u-v}[1-\cos (u-v) \tau]\right. \\
& +\frac{v+w}{v-w}[1-\cos (v-w) \tau]+\frac{w+u}{w-u} \\
& \cdot[1-\cos (w-u) \tau]\} .
\end{aligned}
$$

The maximum gain condition is

$$
\begin{gathered}
(u+v) \sin (u-v)+(v+w) \sin (v-w)+(w+u) \\
\cdot \sin (w-u)=0 .
\end{gathered}
$$

Type B:

$$
\begin{aligned}
f(t)= & \frac{T^{2}}{r^{2}+(u-s)^{2}}\{-\cos u \tau+\cos s \tau \cosh r \tau \\
& \left.-\frac{u-s}{r} \sin s \tau \sinh r \tau\right\} \\
g(t)= & \frac{T^{2}}{r^{2}+(u-s)^{2}}\{-\sin u \tau+\sin s \tau \cosh r \tau \\
& \left.+\frac{u-s}{r} \cos s \tau \sinh r \tau\right\} \\
G(t)= & \frac{2 P}{\left[r^{2}+(u-s)^{2}\right]^{2}}\left\{\frac{s\left[r^{2}+(u-s)^{2}\right]}{r^{2}} \sinh ^{2} r \tau\right. \\
& +\frac{r^{2}+s^{2}-u^{2}}{r} \sin (u-s) \tau \sinh r \tau \\
& +2 u[1-\cos (u-s) \tau \cosh r \tau]\} .
\end{aligned}
$$

When $t \rightarrow \infty$,

$$
G(t) \rightarrow \frac{s P}{2 r^{2}\left[r^{2}+(u-s)^{2}\right]} e^{2 r \tau} .
$$

It is noted that if we change the sign of $(u, v, w)$ in (44) or $(u, s)$ in $(50)$, the gain also changes its sign which seems unphysical. Actually, this is due to the decoupling procedures which result in one sixth-order differential equations from four second-order coupled equations. The choice of the gains overall sign can be done by examining the behavior of the gain at small $t$.

In order to find out the gain at small $t$, we need to determine the lowest order behaviors of $f(t), g(t)$ as well as $\alpha(t)$. Since $f(0)=0, g(0)=0, \alpha(0)=0$, and $\widetilde{E}(0)=1$, we observe from $(18)$ and (21) that

$$
f(t)=\frac{t^{2}}{2}
$$

and

$$
\alpha(t)=\Omega^{\prime} t .
$$

Using (52) in (19), we have

$$
g(t)=-\frac{\Omega^{\prime} t^{3}}{6} .
$$

Substituting (52)-(54) into (20), it is found that

$$
\tilde{E}(t)=1+\frac{b \Omega^{\prime} t^{4}}{12} .
$$

Therefore, the field always grows at the beginning which can be used to determine the correct sign for the gain.

To demonstrate how the field grows in the interaction region, we consider a special example

$$
P \gg \theta_{p}, \theta,
$$




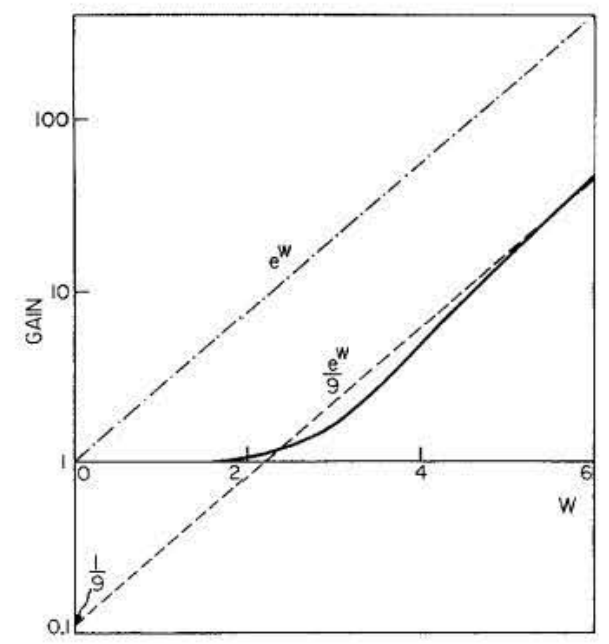

Fig. 4. The gain behavior of a high pumping free-electron laser (or a traveling-wave tube). The gain approaches an exponential growth with a multiplication factor $(1 / 9), W=\sqrt{3} \sqrt[3]{b} t$.

i.e., a strong pumping device. As expected, this is the case of a traveling-wave tube. Equation (33) is then reduced to

$$
\Gamma^{3}= \pm i P^{3} .
$$

Choosing the positive sign in (57), the solutions are

$$
\Gamma=i P ; \quad\left( \pm \frac{\sqrt{3}}{2}-\frac{i}{2}\right) P .
$$

The gain is obtained as

$G(t)=\frac{4}{9}\left\{\cosh ^{2} \frac{\sqrt{3}}{2} P \tau+\cosh \frac{\sqrt{3}}{2} P \tau \cos \frac{3}{2} P \tau-2\right\}$.

Its behavior is plotted in Fig. 4. At the beginning of the interaction region, the gain grows at a very small rate. However, after

$$
t>\frac{2}{\sqrt{3} \sqrt[3]{b}}
$$

the field begins to grow exponentially as

$$
G(t) \rightarrow \frac{1}{9} \exp \sqrt{3} \sqrt[3]{b} t
$$

Although the exponential gain has been obtained from the dispersion equation [6], it might give a wrong impression about the actual behavior of the field. The output field is much smaller than that of a field growing exponentially from the beginning. From (61), it seems that the dominant field grows exponentially from only one-third of the origin field amplitude. This is the first time that a traveling-wave tube has been studied from the single-particle point of view.

\section{CONCLUSION}

We have shown that the Maxwell equations can be incorporated with the Lorentz force equations to give a detailed description of the field variation along the interaction region. Based on the resulting four coupled equations, it is found that the electron beam modulation is described by a three-wave propagation, while the radiation field has a more complicated propagating structure. The field variations are divided into two types: interference-like and exponential-like. The conditions for those two different cases are defined quantitatively in terms of three parameters: frequency mismatch $\theta$, plasma frequency $\theta_{p}$, and pumping strength $P$. An unstable solution is favorable for obtaining high gain if the interaction length is sufficiently long. At high electron densities in an oscillator, the plasma resonance is observed and is due completely to the single-pass gain maximization. A special example shows the advantage of our analysis in the study of a traveling-wave tube which gives the field variation in detail from the beginning.

\section{REFERENCES}

[1] F. A. Hopf, P. Meystre, M. O. Scully, and W. H. Louisell, "Classical theory of a free electron laser," Opt. Commun., vol. 18, pp. 413-416, 1976.

[2] W. B. Colson, "Free electron laser theory," Ph.D. dissertation, Stanford Univ,, Stanford, CA, 1977.

[3] A. Yariv and C. Shih, "Amplification of radiation by relativistic electrons in spatially periodic optical waveguides," Opt. Commun., vol. 24, pp. 233-236, 1978.

[4] W. H. Louisell, J. F. Lam, D. A. Copeland, and W. B. Colson, "Exact classical electron dynamic approach for a free-electron laser amplifier," Phys. Rev. A, vol. 19, pp. 288-300, 1979.

[5] N. M. Kroll, P. Morton, and M. N. Rosenbluth, "Free electron lasers with variable parameter wigglers," JASON Tech. Rep. JSR79-01. Feb. 1980.

[6] N. M. Kroll and W. A. McMullin, "Stimulated emission Irom relativistic electrons passing through a spatially periodic transverse magnetic field," Phys. Rev. A, vol. 17, pp. 300-308, 1978.

[7] A. Gover and Z. Livni, "Operation regimes of Cerenkov-SmithPurcell free electron lasers and T. W. amplifiers," Opt. Commun., vol. 26, pp. $375-380,1978$.

[8] I. B. Bernstein and J. L. Hirshfield, "Amplification on a relativistic electron beam in a spatially periodic transverse magnetic field," Phys. Rev. A, vol. 20, pp. 1661-1670, 1979.

[9] C. Shih, "Theory of longitudinal free electron lasers," Ph.D. dissertation, California Inst. Tech., Pasadena, 1980.

[10] W. B. Colson and S. K. Ride, "The free-electron laser: Maxwell's equations driven by single-particle currents," in Physics of Quantum Electronics, vol. 7, S. F. Jacobs, H. S. Pilloff, M. Sargent III, M. O. Scully, and R. Spitzer, Eds. Reading, MA: AddisonWesley, 1980, pp. 377-414. 
[11] J. R. Pierce, Traveling-Wave Tubes. New York: Van Nostrand, 1950.

[12] C. Shih and A. Yariv, "Single-electron analysis of the spacecharge effect in free-electron lasers," Phys. Rev. A, vol. 22, pp. 2217-2222, 1980.

[13] D. B. McDermott, and T. C. Marshall, "The collective freeelectron laser," in Physics of Quantum Electronics, vol. 7, S. F. Jacobs, H. S. Pilloff, M. Sargent III, M. O. Scully, and R. Spitzer, Eds. Reading, MA: Addison-Wesley, 1980, pp. 509-522.

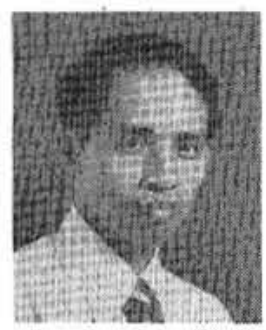

Chun-Ching Shih was born in Taiwan on November 21,1950 . He received the B.S. degree in physics from the National Taiwan University, Taipei, Taiwan, in 1972 and the Ph.D. degree in physics from the California Institute of Technology, Pasadena, CA, in 1980. His thesis research included theoretical studies on longitudinal free electron lasers and the electrooptic effect of single crystals.

From 1979 to 1980 he was a member of the technical staff in R\&D Associates, Marina del Rey, CA. In December 1980 he joined the Plasma Physics Department of TRW, Redondo Beach, CA, where he is currently working in the freeelectron laser program.

Dr. Shih is a member of the American Physical Society and the Optical Society of America.

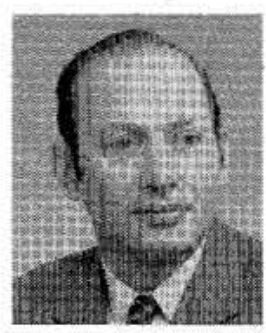

Amnon Yariv (S'56-M'59-F'70) was born in Tel Aviv, Israel, on A pril 13, 1930. He received the B.S., M.S., and Ph.D. degrees, all in electrical engineering, from the University of California, Berkeley, in 1954, 1956, and 1958, respectively.

He was employed by Bell Laboratories, Murray Hill, NJ, in 1959, during the early stages of the laser effort. He then joined the staff of the California Institute of Technology, Pasadena, in 1964, as an Associate Professor of Electrical Engineering, and became a Professor in 1966. He also took part in the discovery of a number of early solid-state laser systems, the formulation of the theory of parametric quantum noise and the prediction of parametric fluorescence, the invention of the technique of mode-locked ultrashort-pulse lasers and FM lasers, the introduction of GaAs and $\mathrm{CdTe}$ as IR electrooptic and window materials, and the proposal and demonstration of semiconductor based integrated optics technology. His present research efforts are in the areas of nonlinear optics, IR electrooptical materials, recombination mechanisms in semiconductors, and thin-film optics. He authored or coauthored numerous papers in professional journals as well as two books: Quantum Electronics (New York: Wiley, 1967) and Introduction to Optical Electronics (New York: Holt. Rinehart and Winston, 1971). He is also an Associate Editor of the IEEE Journal of Quantum Electronics and Optics Communications.

Dr. Yariv is a Fellow of the Optical Society of America, and a member of the American Physical Society and Phi Beta Kappa.

\title{
Application of Particle-in-Cell Simulation in Free-Electron Lasers
}

\author{
THOMAS J. T. KWAN
}

\begin{abstract}
Particle-in-cell simulation of free-electon lasers has contributed significantly to understanding its physics, especially in the nonlinear and multidimensional regimes where analytic theory is rather difficult to formulate. The basic techniques of plasma simulation and the one- and two-dimensional codes used in the simulation of freeelectron lasers are discussed in detail. Important results obtained from simulation are presented. It has been found that waves propagating obliquely with respect to the electron beam are always unstable with appreciable growth rates and, therefore, the efficiency of generation of the on-axis high-frequency electromagnetic wave can be severely degraded. Furthermore, electromagnetic waves with group velocities opposite to the direction of electron beam propagation can be absolutely unstable. Complete disruption of the electron beam and laser oscillation due to the onset of the absolute instability have been observed in simulations. Moreover, computer simulation also shows that the absolute instability can be avoided if the parameters of the freeelectron laser satisfy certain constraints. The dynamics of nonlinear saturation and the potential efficiency of energy extraction are presented in detail.
\end{abstract}

Manuscript received February 3, 1981; revised March 3, 1981. This work was supported by the United States Department of Energy.

The author is with the Applied Theoretical Physics Division, Los Alamos National Laboratory, Los Alamos, NM 87545.

\section{INTRODUCTION}

I $\mathrm{N}$ recent years, there has been a tremendous interest in the concept of free-electron lasers. In the theoretical [1]-[9] and experimental [10]-[12] areas, important advances have been made. More recently, the idea of using a magnetic wiggler field, the wavelength of which is a function of the axial position, appears to be very promising for enhancing the efficiency of energy extraction from the electron beam [13]-[16]. Due to the complexity of the problem, one often has to make some kind of approximations and assumptions in order to make analytic progress. And yet, most of the analytic work is limited to one-dimensional calculation in which all physical variables vary only in one spatial dimension. Thus, some of the important multidimensional effects, such as radial inhomogeniety and off-axis mode production, are ignored. Furthermore, the self-fields produced by the electron beam are often neglected by assuming charge and current neutralization of the electron beam. For such a difficult and complicated problem, particle-in-cell plasma simulation can uniquely provide one-, two-, or even three-dimensional realistic simulation of the 